\title{
Association of $H L A-D R B 1$ amino acid residues with giant cell arteritis: genetic association study, meta-analysis and geo-epidemiological investigation
}

Sarah Louise Mackie', John C. Taylor', Lubna Haroon-Rashid', Stephen Martin', Bhaskar Dasgupta², Andrew Gough ${ }^{3}$, Michael Green ${ }^{4}$, Lesley Hordon ${ }^{5}$, Stephen Jarrett ${ }^{6}$, Colin T. Pease ${ }^{7}$, Jennifer H. Barrett ${ }^{1}$, Richard Watts ${ }^{8+}$, Ann W. Morgan ${ }^{1,9^{*}+}$, UK GCA Consortium and UKRAG Consortium

\begin{abstract}
Introduction: Giant cell arteritis (GCA) is an autoimmune disease commonest in Northern Europe and Scandinavia. Previous studies report various associations with HLA-DRB1*04 and HLA-DRB1*01; HLA-DRB1 alleles show a gradient in population prevalence within Europe. Our aims were (1) to determine which amino acid residues within HLA-DRB1 best explained HLA-DRB1 allele susceptibility and protective effects in GCA, seen in UK data combined in meta-analysis with previously published data, and (2) to determine whether the incidence of GCA in different countries is associated with the population prevalence of the HLA-DRB1 alleles that we identified in our meta-analysis.
\end{abstract}

Methods: GCA patients from the UK GCA Consortium were genotyped by using single-strand oligonucleotide polymerization, allele-specific polymerase chain reaction, and direct sequencing. Meta-analysis was used to compare and combine our results with published data, and public databases were used to identify amino acid residues that may explain observed susceptibility/protective effects. Finally, we determined the relationship of HLA-DRB1*04 population carrier frequency and latitude to GCA incidence reported in different countries.

Results: In our UK data (225 cases and 1378 controls), HLA-DRB ${ }^{*} 04$ carriage was associated with GCA susceptibility (odds ratio $(\mathrm{OR})=2.69, P=1.5 \times 10^{-11}$ ), but $H L A-D R B 1^{*} 01$ was protective (adjusted $\mathrm{OR}=0.55, P=0.0046$ ). In meta-analysis combined with 14 published studies (an additional 691 cases and 4038 controls), protective effects were seen from HLA-DR2, which comprises HLA-DRB $1^{*} 15$ and HLA-DRB $1^{*} 16\left(\mathrm{OR}=0.65, P=8.2 \times 10^{-6}\right)$ and possibly from HLA-DRB $1^{*} 01$ (OR $=0.73, P=0.037)$. GCA incidence ( $n=17$ countries) was associated with population HLA-DRB $1^{*} 04$ allele frequency $\left(P=0.008\right.$; adjusted $R^{2}=0.51$ on univariable analysis, adjusted $R^{2}=0.62$ after also including latitude); latitude also made an independent contribution.

Conclusions: We confirm that HLA-DRB1*04 is a GCA susceptibility allele. The susceptibility data are best explained by amino acid risk residues $\mathrm{V}, \mathrm{H}$, and $\mathrm{H}$ at positions 11,13 , and 33 , contrary to previous suggestions of amino acids in the second hypervariable region. Worldwide, GCA incidence was independently associated both with population frequency of $H L A-D R B 1^{*} 04$ and with latitude itself. We conclude that variation in population $H L A-D R B 1^{*} 04$ frequency may partly explain variations in GCA incidence and that $H L A-D R B 1^{*} 04$ may warrant investigation as a potential prognostic or predictive biomarker.

\footnotetext{
* Correspondence: a.w.morgan@leeds.ac.uk

${ }^{\dagger}$ Equal contributors

'School of Medicine and NIHR-Leeds Biomedical Research Unit, Chapel

Allerton Hospital, Leeds LS7 4SA, West Yorkshire, UK

'Wellcome Trust Brenner Building, St. James's University Hospital, Beckett

Street, Leeds LS9 7TF, West Yorkshire, UK

Full list of author information is available at the end of the article
} 


\section{Introduction}

Genetic association studies are a well-established method for investigating genetic contributions to disease. In rheumatoid arthritis (RA) [1] and small-vessel vasculitis [2], genetically distinct subsets have been identified that have different associations with the major histocompatibility complex (MHC) region that encodes the HLA-DRB1 alleles. Comparison of $H L A-D R B 1$ associations with RA in different ethnic groups helped to support the original "shared epitope" hypothesis of RA susceptibility [3] based on an amino acid risk motif at positions $67-74$ in the third hypervariable region (HVR3) of the class II MHC molecule, encoded by $H L A-D R B 1$. The group of RA "shared epitope" alleles now includes HLA-DRB1*01:01, HLADRB1*04:01, HLA-DRB1*04:04, HLA-DRB1*04:08, and $H L A-D R B 1^{* 10}$ :01; other alleles provide weaker protective effects, additional to the risk effects of the "shared epitope" [4]. Recently, it has been demonstrated that amino acid residues 11 and 13 in the first hypervariable region (HVR1) of class II MHC display the strongest associations with RA susceptibility [5].

Giant cell arteritis (GCA) incidence is highest in populations with Scandinavian ancestry [6-8] and this has led to suggestions that this might be due to genetic factors $[9,10]$. Susceptibility to GCA has been reported to be associated with carriage of $H L A-D R B 1^{*} 04$, but not all studies have shown an association and there are conflicting data as to whether there is an association with specific $H L A-D R B 1^{*} 04$ alleles [11]. Underrepresentation of $H L A-D R B 1 * 01$ in GCA patients from Rochester, Minnesota, led to a suggestion that the risk of GCA may be due to a DRYF motif at positions 28-31 in the second hypervariable region (HVR2) of MHC class II [12], but a Spanish study failed to replicate this finding [13]. To date, however, formal meta-analysis has not yet been performed to determine the major susceptibility and protective $H L A-D R B 1$ alleles. The relative contribution of genetic and environmental factors as an explanation for geographical differences in GCA incidence also remains disputed $[6,7]$. When genetic diversity within Europe is subjected to principal component analysis, the MHC is one of several genetic regions that are strongly associated with a component that runs along a north-south gradient from Norway/Sweden to Spain [14]. We therefore hypothesised that variations in the frequency of $H L A-D R B 1$ GCA susceptibility alleles may partly explain geographical variations in GCA incidence.

Here, we report new GCA susceptibility data, combine these with the published data using meta-analysis, and propose a new hypothesis regarding a possible amino acid GCA 11-13-33 risk motif in HVR1 and HVR2 of class II MHC. This hypothesis fits the observed data better than previously proposed models.

\section{Methods}

\section{Patients}

The UK GCA Consortium was designed to support genetic association studies. Investigators, all experienced rheumatologists, recruited cases with a firm clinical diagnosis of GCA, based on all available information. Recruitment was retrospective. A positive temporal artery biopsy was not required as it was not always undertaken in classic presentations or could not be performed within an optimal time window or both. In some centres, the erythrocyte sedimentation rate was unavailable and so fulfilment of the 1990 American College of Rheumatology (ACR) criteria [15], which should not be used for clinical diagnosis of GCA [16], was not a requirement for inclusion. Clinical data on a subset of this cohort have already been published [17]. In this analysis, we included all patients who agreed to give a blood sample for genetic studies up to 2012 and where a sample was available. Written informed consent was provided by all patients, and the study was approved by the York Research Ethics Committee (reference 05/Q1108/28).

\section{DNA extraction and genotyping}

DNA was extracted from peripheral blood. HLA-DRB1 genotyping was performed by either single-stranded oligonucleotide polymerisation [18] or allele-specific polymerase chain reactions (standard primer sequences (HLA DRBplus Typing Kit, Amersham Biosciences, now part of GE Healthcare, Little Chalfont, UK), except for the forward primer of $H L A-D R B 1^{*} 10$ which was redesigned as 5'-GCG GTT GCT GGA AAG ACG CG-3'). Direct sequencing was also performed to enable fourdigit genotyping of $H L A-D R B 1 * 04$ subtypes [18] because of previous reports of a $H L A-D R B 1^{*} 04$ association of GCA at the two-digit level. The HaplotypeViewer program was developed to facilitate rapid four-digit genotyping from sequence electropherograms and is freely available [19].

\section{Analysis of genotyping data}

Control data from the UK Rheumatoid Arthritis Genetics (UKRAG) Consortium were used for this analysis. Initial logistic regression analyses were undertaken by assuming additive genetic models to estimate the effect of each potential susceptibility/protective allele. Adjustments for genetic effects already proposed in the literature (HLA$D R B 1 * 04)$ were also performed.

\section{Meta-analysis of giant cell arteritis susceptibility data}

To identify case-control studies of HLA-DRB1 association with GCA susceptibility, a literature search was conducted in PubMed, without language restriction, by using the terms "HLA" and "(giant cell arteritis) OR (temporal arteritis)". Reference lists of studies identified 
were also scanned. Publications were included if they provided sufficient detail on cases and controls to perform a meta-analysis. Where there were multiple publications with overlapping datasets, the report with the most complete dataset was chosen. Meta-analysis of the published summary carrier frequency data was performed (i.e., assuming a dominant mode of inheritance) because allele frequency and individual-level patient data were mostly unavailable from the authors of the studies. A random-effects model was used; the overall estimate was calculated by using as weights $1 /\left(v_{\mathrm{i}}+\tau\right)$, where $v_{\mathrm{i}}$ is the variance of the estimated effect from the $i$ th study and $\tau$ is the estimated between-study variance [20].

\section{Worldwide giant cell arteritis incidence in relation to $H L A-D R B 1 * 04$ population carrier frequencies}

To identify reports of the incidence of GCA in different countries, a second literature search in PubMed was conducted with combinations of the medical subject heading terms "giant cell arteritis", "temporal arteritis", and "epidemiology". Hand-searching was also performed in the reference lists of retrieved articles, review articles, and textbooks. Studies were included if they were available in full-text and included an estimate of the annual incidence, time period of the study, method of case definition, population studied, and geographical location of the study. Where necessary, the incidence figure was recalculated as number of new cases per 100,000 of the over-50 population per year. Studies that appeared to report duplicate or overlapping populations were excluded. Studies completing recruitment before 1980 were excluded in case of time trends in the incidence of GCA and because the quality of the reporting was generally lower for the older studies. Where more than one report existed for a single country (unless in ethnically distinct populations), the one with a later average period of recruitment was preferred. Where a single report included two separate sub-studies (regions or time periods), a weighted mean of the two sub-studies was used to arrive at an overall incidence figure.

We then sought data on ethnically matched $H L A$ $D R B 1$ population allele frequencies at the two- and fourdigit levels for each geographical region identified in the second literature search. We considered $H L A-D R B 1^{*} 04$ alleles and those identified as being potential susceptibility/protective alleles in our own UK dataset. Methods have been reported elsewhere [21]; briefly, we first consulted the Allele Frequency Net Database [22] and then, if necessary, Ovid Medline and Embase. Carrier frequencies for control populations were converted to estimated allele frequencies by using the Hardy-Weinberg equation. Finally, the following predetermined rule was used to generate an estimate of population $H L A-D R B 1$ allele frequencies: reports with over 500 (four-digit typing) or
1000 (two-digit typing) controls were identified and a weighted mean calculated. In the absence of large studies, studies with more than 100 (four-digit typing) or more than 200 (two-digit typing) were identified and a weighted mean calculated. Determination of geographical latitude and linear regression analysis were performed as previously described [21].

\section{Development of amino acid risk motif model}

After determination of susceptibility and protective HLA-DRB1 alleles, amino acid residues in HVR1, HVR2, and HVR3 were obtained from the IMGT (International ImMunoGeneTics Information System) database [23], accessed 31 January 2012). For samples with only twodigit typing, we estimated amino acid residues on the basis of geographically relevant population frequencies of the four-digit subtypes from the Allele Frequency Net Database [22], assigning a probability to residues when they varied within the four-digit subtypes (only necessary at positions $28,32,37,67,70,71$, and 74 ). For each of these, the expected misclassification rate when assigned by using population frequencies is less than 1 $\%$, apart from positions $67(2 \%)$ and 71 (3\%). For each polymorphic position, samples were assigned a dosage (i.e., the expected number of copies) for each residue. Logistic regression was then used to test for association at each position separately, and degrees of freedom were equal to one less than the number of distinct residues. For the most significant positions, forward stepwise regression was used to identify the residues associated with disease risk at that position.

We used population HLA-DRB1 frequencies for inferring amino acid residues in both cases and controls. Under the null hypothesis of no association, the frequencies would be the same, and any bias introduced by using population frequencies for cases would be toward the null. Using $H L A-D R B 1$ four-digit frequencies that have been observed in patients with GCA to infer the amino acid residues in the GCA cases could lead to a biased analysis with inflated false-positive rate.

Results that reach a nominal significance level of 0.05 are highlighted. For the exploratory hypotheses, these should be interpreted in the light of multiple testing. Analyses were performed in SPSS 15 (IBM Corporation, Armonk, NY, USA) and Stata SE (StataCorp LP, College Station, TX, USA).

\section{Results}

\section{Patients}

Two hundred twenty-five patients with GCA from 7 UK centres consented to analysis of genetic material for this study (125 from Leeds hospitals, including 38 from Otley; 33 from Harrogate; 23 from Southend; 17 from York; 16 from Dewsbury; 10 from Pontefract; and one 
from Ipswich). Their demographics and disease characteristics, including fulfilment of 1990 ACR criteria, are shown in Table 1 . Of the 183 temporal artery biopsies performed, 140 (77\%) were positive. Patients were all European Caucasian.

\section{Analysis of genotyping data}

Allele frequencies in cases and 1378 UKRAG controls are shown in Table 2 with per-allele odds ratios with and without adjustment for $H L A-D R B 1 * 04$, the previously proposed susceptibility allele. Initial analysis was performed at the two-digit level. Four-digit analysis was also performed for the common "04 subtypes, but statistical analysis was not performed on the rarer "04 subtypes.

In 225 patients with GCA and 1378 controls in the novel UK cohort, a susceptibility effect of $H L A-D R B 1 * 04$ carriage was confirmed (odds ratio (OR) 2.69, $95 \%$ confidence interval (CI) 2.02 to $3.58, P=1.5 \times 10^{-11}$ ) (Table 3). Possible protective effects from $H L A-D R B 1^{*} 01$ and $H L A-D R B 1 * 15$ were noted, but only HLA-DRB1*01 retained significance after adjusting for $H L A-D R B 1 * 04$ (Table 2). The data were consistent with a dominant effect of HLA-DRB1*04 (OR for one copy $2.78,95 \% \mathrm{CI}$ 2.07 to 3.72 , OR for two copies 1.94, $95 \%$ CI 0.95 to 3.96 , compared with no copies).

The effect sizes for $H L A-D R B 1$ *04 carriage were similar when restricting analyses to biopsy-positive GCA cases $\left(\mathrm{OR}=2.83,1.99\right.$ to $\left.4.03, P=7.7 \times 10^{-9}\right)$; the number of biopsy-negative GCA cases was too small for a separate analysis.

Meta-analysis of giant cell arteritis susceptibility data

Meta-analysis of previously published data from 14 studies (691 cases, 4038 controls; Table 3) gave ORs of 2.45 $\left(P=9.2 \times 10^{-24}\right), 0.78(P=0.11)$, and $0.71(P=0.0019)$ for HLA-DRB1 *04, "01, and "02, respectively ("02 is now reclassified as "15 and "16). There was more heterogeneity noted for some alleles than for others; the $H L A$ $D R B 1 * 04$ meta-analysis had an $\mathrm{I}^{2}$ statistic of $0 \%$ whereas the meta-analysis for $H L A-D R B 1^{*} 01$ had an $\mathrm{I}^{2}$ statistic of $39.4 \%$. When our UK data was included, the metaanalysis still demonstrated protective effects for $H L A$ DRB1*01 and HLA-DRB1*02 $(P=0.037$ and $P=8.2 \times 10$ ${ }^{-6}$, respectively) (Table 3$)$. In the six published articles with information on ethnicity, cases and controls were always stated to be either "white" or "Caucasian". Therefore, subgroup meta-analysis by ethnic group was not possible.

\section{Worldwide giant cell arteritis incidence in relation to HLA-DRB $1 * 04$ population carrier frequencies}

Reliable population $H L A-D R B 1$ data were not always available, notably for small, native tribes of Alaska and Saskatoon, where extrapolation from small and physically or genetically (or both) isolated communities was felt to be unwarranted. Table 4 summarises the GCA incidence articles included, together with the estimated population allele frequencies and the number of individuals on which these estimates are based. Substantial clinical heterogeneity was identified in the GCA incidence studies, including variations in the methods used to identify GCA cases and confirm GCA diagnosis.

At the two-digit level (Table 4 and Fig. 1a and b), 17 studies were included in the analysis of worldwide GCA incidence in relation to population $H L A-D R B 1$ allele frequencies. In view of our meta-analysis findings, we extracted data for $H L A-D R B 1^{*} 04, H L A-D R B 1^{*} 01$, and $H L A$ $D R B 1^{*} 15$ population frequencies (Table 4). The majority of these were from Europe and the Mediterranean area. Within this small dataset, HLA-DRB1*15 was more common in the general population at more northerly latitudes

Table 1 Patient characteristics

\begin{tabular}{lll}
\hline Patient characteristic & Value (\% of those with data available) & Number with data available \\
\hline Female & $161(72 \%)$ & 225 \\
Median age at onset of GCA (range), years & $72(50-94)$ & 207 \\
Biopsy-positive & $140(77 \%)$ & 183 (number of cases biopsied) \\
Median erythrocyte sedimentation rate at onset of GCA (range), mm/h & $70(5-150)$ & 100 \\
Median plasma viscosity at onset of GCA (range), mPa s & $1.94(1.53-2.65)$ & 120 \\
Median C-reactive protein at onset of GCA (range), mg/l & $62.5(<5-344)$ & 154 \\
Either ACR 1990 criteria for GCA fulfilled or biopsy-positive & $207(92 \%)$ & 225 \\
Headache & $184(90 \%)$ & 204 \\
Abnormal temporal artery as defined by ACR 1990 criteria & $140(67 \%)$ & 204 \\
Jaw claudication & $119(57 \%)$ & 210 \\
Visual or neurological features & $116(56 \%)$ & 207
\end{tabular}

Owing to the retrospective recruitment of cases, contemporaneously recorded data on features of giant cell arteritis (GCA) at presentation were not always recorded in the medical notes. For this reason, in 24 cases, fulfilment of American College of Rheumatology (ACR) criteria could not be documented. Six of these were biopsy-proven. In some recruiting centres, plasma viscosity or C-reactive protein was measured instead of erythrocyte sedimentation rate 
Table 2 Allele frequencies and per-allele odds ratios in giant cell arteritis cases and controls

\begin{tabular}{|c|c|c|c|c|c|c|c|}
\hline HLA-DRB1 allele & $\begin{array}{l}\text { Allele frequency } \\
\text { in controls, N (\%) }\end{array}$ & $\begin{array}{l}\text { Allele frequency } \\
\text { in cases, N (\%) }\end{array}$ & $\begin{array}{l}\text { Allele frequency in } \\
\text { biopsy-positive cases, N (\%) }\end{array}$ & Unadjusted OR (95 \% Cl) & $P$ value & $\begin{array}{l}\text { OR adjusted } \\
\text { for }{ }^{*} 04(95 \% \mathrm{Cl})\end{array}$ & $P$ value \\
\hline${ }^{*} 01$ & $341(12.4)$ & $27(6.0)$ & $12(4.3)$ & $0.46(0.31,0.69)$ & $1.6 \times 10^{-4}$ & $0.55(0.37,0.83)$ & 0.0046 \\
\hline${ }^{*} 03$ & $438(15.9)$ & $75(16.7)$ & $41(14.5)$ & $1.06(0.81,1.39)$ & 0.68 & $1.31(0.99,1.74)$ & 0.061 \\
\hline$*^{*} 04$ & $503(18.3)$ & $138(30.7)$ & $89(31.6)$ & $2.03(1.61,2.56)$ & $1.7 \times 10^{-9}$ & - & - \\
\hline$*^{*} 04: 01$ & $304(11.0)$ & $83(18.4)$ & $54(19.1)$ & $1.87(1.42,2.46)$ & $8.2 \times 10^{-6}$ & - & - \\
\hline${ }^{*} 04: 02$ & $10(0.4)$ & $2(0.4)$ & $1(0.4)$ & $1.29(0.64,2.59)^{*}$ & $0.48^{*}$ & - & - \\
\hline *04:03 & $38(1.4)$ & $8(1.8)$ & $7(2.5)$ & & & & \\
\hline$* 04: 04 / 04: 08$ & $151(5.5)$ & $45(10.0)$ & $27(9.6)$ & $1.86(1.32,2.63)$ & $3.7 \times 10^{-4}$ & - & - \\
\hline${ }^{*} 07$ & $393(14.3)$ & $57(12.7)$ & $34(12.1)$ & $0.88(0.65,1.17)$ & 0.37 & $1.03(0.76,1.39)$ & 0.85 \\
\hline *08 & $78(2.8)$ & $8(1.8)$ & $4(1.4)$ & $0.62(0.30,1.29)$ & 0.20 & $0.75(0.36,1.57)$ & 0.45 \\
\hline *09 & $30(1.1)$ & $5(1.1)$ & $5(1.8)$ & $1.02(0.39,2.66)$ & 0.97 & $1.19(0.45,3.12)$ & 0.73 \\
\hline$* 10$ & $21(0.8)$ & $0(0.0)$ & $0(0.0)$ & - & - & - & \\
\hline$* 11$ & $179(6.5)$ & $32(7.1)$ & $20(7.1)$ & $1.09(0.75,1.59)$ & 0.64 & $1.25(0.86,1.83)$ & 0.25 \\
\hline$* 12$ & $49(1.8)$ & $10(2.2)$ & $6(2.1)$ & $1.24(0.64,2.42)$ & 0.53 & $1.60(0.81,3.14)$ & 0.18 \\
\hline$* 13$ & $222(8.1)$ & $41(9.1)$ & $32(11.3)$ & $1.14(0.81,1.62)$ & 0.45 & $1.35(0.95,1.93)$ & 0.098 \\
\hline$* 14$ & $60(2.2)$ & $10(2.2)$ & $5(1.7)$ & $1.02(0.52,2.00)$ & 0.95 & $1.29(0.65,2.56)$ & 0.46 \\
\hline *15 & $421(15.3)$ & $45(10.0)$ & $32(11.3)$ & $0.63(0.46,0.87)$ & 0.0047 & $0.75(0.54,1.04)$ & 0.085 \\
\hline$* 16$ & $21(0.8)$ & $2(0.4)$ & $2(0.7)$ & $0.58(0.13,2.49)$ & 0.46 & $0.67(0.16,2.92)$ & 0.60 \\
\hline
\end{tabular}

$N$ number of copies of allele seen in 1378 controls or 225 cases, OR odds ratio, $\mathrm{Cl}$ confidence interval

*denotes where $H L A-D R B 1^{*} 04: 02$ and ${ }^{*} 04: 03$ categories were combined because of low numbers

$(r=0.52, P=0.038)$ whereas no significant association with latitude was seen for population $H L A-D R B 1^{*} 04$ or HLA-DRB1*01 ( $r=0.47, P=0.057 ; r=0.39, P=0.133)$.

Predictors of GCA incidence in univariable analyses were $H L A-D R B 1 * 04$ population allele frequency $(P=$ 0.001 , adjusted $\left.R^{2}=0.51\right)$ and latitude $(P=0.004$, adjusted $\left.\mathrm{R}^{2}=0.40\right)$, whereas $H L A-D R B 1 * 15$ was nonsignificant. In multivariable analysis, both were significant predictors and each made independent contributions to the explanatory power of the model (HLA-DRB1*04, $P=$ 0.008 ; latitude, $P=0.036$; adjusted $\mathrm{R}^{2}$ of the model $=$ 0.62). $H L A-D R B 1 * 15$ made no additional contribution to the explanatory power of the model.

Development of model with 11-13-33 amino acid risk motif Tests for association showed that the most significant position was at $13\left(P=1.2 \times 10^{-9}\right)$, followed by $11,33,37$, and 9 (Fig. 1c). At position 13, the most significant residue was $\mathrm{H}\left(\mathrm{OR}=2.11,95 \% \mathrm{CI} 1.61\right.$ to $2.77, P=5.5 \times 10^{-8}$, equivalent to $\mathrm{H}$ at 33 and also to the 04 allele). However, stepwise regression found additional contributions from residues $\mathrm{S}(\mathrm{OR}=1.38,95 \% \mathrm{CI} 1.07$ to $1.77, P=0.014)$ and $\mathrm{F}(\mathrm{OR}=0.66,95 \% \mathrm{CI} 0.44$ to $0.99, P=0.038)$ (Table 5). Similarly, multiple residues were found at positions 11 and 37. There is very strong linkage disequilibrium in this region, and so many of these residues at different positions almost always occur together, as illustrated in Additional file 1. For example, we did not have the power to distinguish between the risk effects of $\mathrm{V}, \mathrm{H}$, and $\mathrm{H}$ at positions 11,13 , and 33 , respectively, since the *10 allele, which differs at residue 13 , is very rare. The previously proposed DYF motif (positions 28, 30, and 31) in HVR2 (OR $=1.54,95 \% \mathrm{CI} 1.21$ to $1.96, P=0.00038$ ) did not explain the observed data as well as simple $H L A$ $D R B 1^{*} 04$ carriage. Similarly, variation in amino acid residues within HVR3 is unlikely to explain the observed GCA susceptibility data (Fig. 1c), especially since the other alleles comprising the "RA shared epitope" were not overrepresented in GCA.

\section{Discussion}

In this study, which includes both new UK data and the first formal meta-analysis of published data on HLA$D R B 1$ associations of GCA, we not only confirm a strong association of GCA with $H L A-D R B 1 * 04$ allele carriage, including within our own UK data, but also identify possible protective effects of $H L A-D R B 1 * 01$ and $H L A-D R B 1 * 15$, supported by the meta-analysis of previous studies. We were able to impute amino acid residues quite reliably from published allele frequencies, enabling us to analyse amino acid residues even though four-digit typing was not available for every $H L A-D R B 1$ allele. Based on this, it was the amino acid residues 11,13 , and 33 in the first and second hypervariable regions that best 
Table 3 Meta-analysis of HLA-DRB1 giant cell arteritis associations in the literature

\begin{tabular}{|c|c|c|c|c|c|c|c|c|c|c|}
\hline \multirow[t]{2}{*}{ HLA-DRB1 allele(s) } & \multicolumn{2}{|l|}{ UK GCA Cohort } & \multicolumn{4}{|l|}{ Published data only } & \multicolumn{4}{|c|}{ Combination of data in this report with published data } \\
\hline & OR $(95 \%$ Cl) for carriage of allele & $P$ value & Number of studies & $\begin{array}{l}\text { OR ( } 95 \% \text { Cl) for } \\
\text { carriage of allele }\end{array}$ & $\begin{array}{l}\text { Meta-analysis } \\
P \text { value }\end{array}$ & $\begin{array}{l}\text { Heterogeneity } \\
I^{2} \text { statistic (\%) }\end{array}$ & $\begin{array}{l}\text { Number of } \\
\text { studies }\end{array}$ & $\begin{array}{l}\text { OR (95 \% Cl) for } \\
\text { carriage of allele }\end{array}$ & $\begin{array}{l}\text { Meta-analysis } \\
P \text { value }\end{array}$ & $\begin{array}{l}\text { Heterogeneity } \\
I^{2} \text { statistic (\%) }\end{array}$ \\
\hline *01 & $0.46(0.30,0.70)$ & 0.00033 & 14 & $0.78(0.57,1.06)$ & 0.11 & 39.4 & 15 & $0.73(0.54,0.98)$ & 0.037 & 48.1 \\
\hline DR2 (*15 and *16) & $0.53(0.37,0.76)$ & 0.00050 & 13 & $0.71(0.57,0.88)$ & 0.0019 & 0 & 14 & $0.65(0.54,0.79)$ & $8.2 \times 10^{-6}$ & 0 \\
\hline *03 & $1.12(0.83,1.52)$ & 0.47 & 14 & $1.02(0.81,1.28)$ & 0.87 & 10.0 & 15 & $1.05(0.88,1.27)$ & 0.58 & 4.2 \\
\hline *04 & $2.69(2.02,3.58)$ & $1.5 \times 10^{-11}$ & 15 & $2.45(2.06,2.92)$ & $9.2 \times 10^{-24}$ & 0 & 16 & $2.51(2.16,2.92)$ & $1.1 \times 10^{-33}$ & 0 \\
\hline${ }^{*} 05\left({ }^{*} 11\right.$ and $\left.{ }^{* 12}\right)$ & $1.21(0.83,1.75)$ & 0.32 & 9 & $0.85(0.65,1.12)$ & 0.24 & 0 & 10 & $0.96(0.77,1.20)$ & 0.72 & 0 \\
\hline${ }^{*} 06\left({ }^{*} 13\right.$ and $\left.{ }^{*} 14\right)$ & $1.11(0.78,1.57)$ & 0.56 & 10 & $0.69(0.48,0.99)$ & 0.042 & 27.1 & 11 & $0.76(0.55,1.06)$ & 0.11 & 40.6 \\
\hline${ }^{*} 07$ & $0.92(0.66,1.28)$ & 0.63 & 14 & $1.14(0.81,1.60)$ & 0.45 & 65.0 & 15 & $1.12(0.83,1.50)$ & 0.47 & 63.4 \\
\hline$* 11$ & $1.18(0.78,1.79)$ & 0.43 & 6 & $0.90(0.65,1.26)$ & 0.55 & 6.9 & 7 & $1.00(0.77,1.30)$ & 0.98 & 5.1 \\
\hline${ }^{* 13}$ & $1.15(0.79,1.68)$ & 0.47 & 5 & $0.68(0.47,1.00)$ & 0.050 & 0 & 6 & $0.89(0.68,1.16)$ & 0.39 & 0 \\
\hline
\end{tabular}

GCA giant cell arteritis, $O R$ odds ratio, $C l$ confidence interval

Insufficient data were present for $H L A-D R B 1 * 08,{ }^{*} 09, * 10, * 12,{ }^{*} 14,{ }^{*} 15$ and ${ }^{*} 16$ to perform meta-analysis. Published studies included in this meta-analysis for each allele were $H L A-D R B 1 * 01 /{ }^{*} 03 /{ }^{*} 07$ [13, 36-48], HLA-DRB $7^{*} 02[13,36-46,48], H L A-D R B 1^{*} 05[13,36-39,41-43,46], H L A-D R B 1^{*} 06[13,36-39,41-44,46], H L A-D R B 1^{*} 11[13,40,44,45,47,48]$, and HLA-DRB1*13 [13, 40, 45, 47, 48] 
Table 4 Incidence of giant cell arteritis and population HLA-DRB1 allele frequencies in different countries

\begin{tabular}{|c|c|c|c|c|c|c|c|c|c|}
\hline \multicolumn{5}{|l|}{ GCA incidence study } & \multicolumn{5}{|c|}{ HLA-DRB1 allele frequency and references (if multiple references given, the allele frequency is the weighted mean) } \\
\hline $\begin{array}{l}\text { City/region, } \\
\text { country, reference }\end{array}$ & $\begin{array}{l}\text { Dates, study } \\
\text { design }\end{array}$ & GCA criteria & $\begin{array}{l}\text { GCA incidence, } \\
\text { per 100,000 per } \\
\text { year in over-50s }\end{array}$ & $\begin{array}{l}\text { Latitude } \\
\text { (degrees N) }\end{array}$ & ${ }^{*} 04$ & ${ }^{*} 01$ & *15 & ${ }^{*} 04: 01$ & *04:04 \\
\hline $\begin{array}{l}\text { W Nyland, } \\
\text { Finland [49] }\end{array}$ & $\begin{array}{l}\text { 1987-88, } \\
\text { prospective }\end{array}$ & Biopsy, clinical & 26.2 & 61.5 & $0.147(n=1157[50])$ & $0.183(n=1157[50])$ & $0.144(n=1157[50])$ & $\begin{array}{l}0.080 \\
(n=1157[50])\end{array}$ & $0.037(n=1157[50])$ \\
\hline $\begin{array}{l}\text { Goteborg, } \\
\text { Sweden [51] }\end{array}$ & $\begin{array}{l}\text { 1976-95, } \\
\text { retrospective }\end{array}$ & Biopsy, clinical & 22.2 & 57.7 & $\begin{array}{l}0.195(\mathrm{n}=1347[22], \\
1366[52], 1209[53] \\
1191[54])\end{array}$ & $\begin{array}{l}0.105(n=1347[22], \\
1366[52], 1209[53] \\
1191[54])\end{array}$ & $\begin{array}{l}0.159(n=1347[22], \\
1366[52], 1209[53], \\
1191[54])\end{array}$ & $\begin{array}{l}0.137(n=934 \\
{[55], 1763[56],} \\
1191[54])\end{array}$ & $\begin{array}{l}0.057(n=934[55] \\
1763[56], 1191[54])\end{array}$ \\
\hline $\begin{array}{l}\text { Reggio Emilia, } \\
\text { Italy [57] }\end{array}$ & $\begin{array}{l}\text { 1980-88, } \\
\text { retrospective }\end{array}$ & Biopsy & 6.9 & 44.7 & $\begin{array}{l}0.072(n=57,345[58] \\
4080[22], 2054[22] \\
1992[59])\end{array}$ & $\begin{array}{l}0.089(n=57,345[58] \\
4080[22], 2054[22] \\
1992[59])\end{array}$ & $\begin{array}{l}0.068(n=57,345[58], \\
2054[22], 1992[59])\end{array}$ & $\begin{array}{l}0.017 \\
(n=57,345[58])\end{array}$ & $0.012(n=57345[58])$ \\
\hline $\begin{array}{l}\text { Lugo, NW } \\
\text { Spain [60] }\end{array}$ & $\begin{array}{l}\text { 1981-98, } \\
\text { retrospective }\end{array}$ & Biopsy & 10.2 & 43.0 & $\begin{array}{l}0.129(n=1940[61] \\
1088[62])\end{array}$ & $\begin{array}{l}0.103(n=1940[61] \\
1088[62])\end{array}$ & $\begin{array}{l}0.104(n=1940 \\
[61], 1088[62])\end{array}$ & $\begin{array}{l}0.053 \\
(n=145[22])\end{array}$ & $0.0207(n=145[22])$ \\
\hline Denmark [63] & $\begin{array}{l}\text { 1982-94, } \\
\text { prospective }\end{array}$ & Biopsy & 20.4 & 56.5 & $0.180(n=562[64])$ & $0.101(n=562[64])$ & $0.157(n=562[64])$ & $\begin{array}{l}0.176 \\
(n=55[22])\end{array}$ & $0.001(n=55[22])$ \\
\hline Iceland [65] & $\begin{array}{l}\text { 1984-90, } \\
\text { retrospective }\end{array}$ & ACR 1990 criteria & 27.0 & 64.7 & $0.170(n=172[66])$ & $0.040(n=172[66])$ & $0.020(n=172[66])$ & No data & No data \\
\hline $\begin{array}{l}\text { Alesund and } \\
\text { Bodo, Norway [67] }\end{array}$ & $\begin{array}{l}\text { 1992-6, } \\
\text { retrospective, } \\
\text { hospital }\end{array}$ & $\begin{array}{l}\text { Biopsy/ACR } \\
1990 \text { criteria }\end{array}$ & 32.4 & 64.9 & $\begin{array}{l}0.225(n=576[22], \\
630[68], 368[69], \\
898[55])\end{array}$ & $\begin{array}{l}0.117(n=576[22] \\
630[68], 898[55])\end{array}$ & $\begin{array}{l}0.153(n=576[22] \\
630[68], 368[69] \\
898[55])\end{array}$ & $\begin{array}{l}0.117 \\
(n=898[55])\end{array}$ & $0.067(n=898[55])$ \\
\hline $\begin{array}{l}\text { Schleswig } \\
\text { Holstein, North } \\
\text { Germany [70] }\end{array}$ & $\begin{array}{l}\text { 1998-2002, } \\
\text { prospective, } \\
\text { population }\end{array}$ & $\begin{array}{l}\text { Chapel Hill } \\
\text { consensus criteria }\end{array}$ & 3.2 & 54.2 & $\begin{array}{l}0.140(n=11,407[22], \\
8862[22], 4251[71])\end{array}$ & $\begin{array}{l}0.111(n=11,407[22], \\
8862[22], 4251[71])\end{array}$ & $\begin{array}{l}0.141(n=11,407 \\
{[22], 8862[22],} \\
4251[71])\end{array}$ & $\begin{array}{l}0.081 \\
(n=8862[22])\end{array}$ & $0.024(n=8862[22])$ \\
\hline Vilnius, Lithuania [72] & $\begin{array}{l}\text { 1990-9, } \\
\text { prospective, } \\
\text { hospital }\end{array}$ & ACR 1990 criteria & 0.7 & 54.7 & $0.078(n=134[73])$ & $0.111(n=134[73])$ & No data & No data & No data \\
\hline Jerusalem [74] & $\begin{array}{l}\text { 1980-2004, } \\
\text { retrospective }\end{array}$ & $\begin{array}{l}\text { Biopsy + ACR } \\
1990 \text { criteria }\end{array}$ & 11.3 & 31.8 & $0.170(n=23000[22])$ & $0.083(n=23,000[22])$ & $0.070(n=23,000[22])$ & $\begin{array}{l}0.019 \\
(n=132[75])\end{array}$ & $0.015(n=132[75])$ \\
\hline $\begin{array}{l}\text { Sephardic Jews, } \\
\text { Israel [76] }\end{array}$ & $\begin{array}{l}\text { 1980-91, } \\
\text { retrospective }\end{array}$ & Biopsy & 10.2 & 31.8 & $0.112(n=293[77])$ & $0.038(n=293[77])$ & $0.087(n=293[77])$ & $\begin{array}{l}0.000 \\
(n=293[77])\end{array}$ & $0.009(n=293[77])$ \\
\hline UK [28] & $\begin{array}{l}\text { 1990-2001, } \\
\text { prospective }\end{array}$ & Clinical & 22.0 & 51.5 & $\begin{array}{l}0.204(n=39,979 \\
[22], 1238[78])\end{array}$ & $\begin{array}{l}0.110(n=39,979 \\
[22], 1238[78])\end{array}$ & $\begin{array}{l}0.143(n=39,979 \\
[22], 1238[78])\end{array}$ & $\begin{array}{l}0.116 \\
(n=298[22])\end{array}$ & $0.047(n=298[22])$ \\
\hline Erdirne, Turkey [79] & $\begin{array}{l}\text { 2003-9, } \\
\text { retrospective }\end{array}$ & Clinical & 1.1 & 41.7 & $\begin{array}{l}0.128(n=250[22] \\
253[80], 250[81])\end{array}$ & $\begin{array}{l}0.047(n=250[22] \\
253[80], 250[81])\end{array}$ & $\begin{array}{l}0.082(n=250[22] \\
253[80], 250[81])\end{array}$ & $\begin{array}{l}0.014(n=110 \\
[82], 250[81])\end{array}$ & $\begin{array}{l}0.019(n=110[82] \\
250[81])\end{array}$ \\
\hline \multirow[t]{2}{*}{$\begin{array}{l}\text { Olmsted County, } \\
\text { MN, USA [83] }\end{array}$} & $\begin{array}{l}\text { 2000-2004, } \\
\text { prospective, } \\
\text { population }\end{array}$ & ACR 1990 & 18.9 & 44.0 & $0.156(n=339[22])$ & $0.097(n=339[22])$ & $0.124(n=339[22])$ & $\begin{array}{l}0.091 \\
(n=339[22])\end{array}$ & $0.040(n=339[22])$ \\
\hline & & Biopsy/clinical & 2.2 & 35.2 & & & & & $0.031(n=61,655[22])$ \\
\hline
\end{tabular}


Table 4 Incidence of giant cell arteritis and population HLA-DRB1 allele frequencies in different countries (Continued)

\begin{tabular}{|c|c|c|c|c|c|c|c|c|c|}
\hline $\begin{array}{l}\text { White, Memphis, } \\
\text { TN, USA [84] }\end{array}$ & $\begin{array}{l}\text { 1971-1980, } \\
\text { retrospective }\end{array}$ & & & & $\begin{array}{l}0.156 \\
(n=61,655[22])\end{array}$ & $\begin{array}{l}0.110 \\
(n=61,655[22])\end{array}$ & $\begin{array}{l}0.133 \\
(n=61,655[22])\end{array}$ & $\begin{array}{l}0.091 \\
(n=61,655[22])\end{array}$ & \\
\hline $\begin{array}{l}\text { Black, Memphis, } \\
\text { TN, USA [84] }\end{array}$ & $\begin{array}{l}\text { 1971-1980, } \\
\text { retrospective }\end{array}$ & Biopsy/clinical & 0.4 & 35.2 & $0.060(n=34[85])$ & No data & $0.210(n=34[85])$ & No data & No data \\
\hline $\begin{array}{l}\text { Europeans, } \\
\text { Saskatoon, Canada [86] }\end{array}$ & $\begin{array}{l}\text { 1998-2003, } \\
\text { prospective }\end{array}$ & Biopsy & 9.3 & 52.1 & $0.178(n=415[87])$ & $0.092(n=415[87])$ & $0.147(n=415[87])$ & $\begin{array}{l}0.097 \\
(n=216[88])\end{array}$ & $0.051(n=216[88])$ \\
\hline
\end{tabular}

ACR American Coll

Giant cell arteritis (GCA) incidence in Finland was taken as weighted mean of the two sub-studies reported in the article cited. Allele frequencies are the weighted mean of the allele frequencies reported in the studies cited (see Methods for details of how these were selected). For brevity, where the data were taken from the allelefrequencies.net website, the main citation for the website is given [22]; searching allelefrequencies.net on the country, sample size, and human leukocyte antigen (HLA) allele in question will give the allele frequencies used in this table 


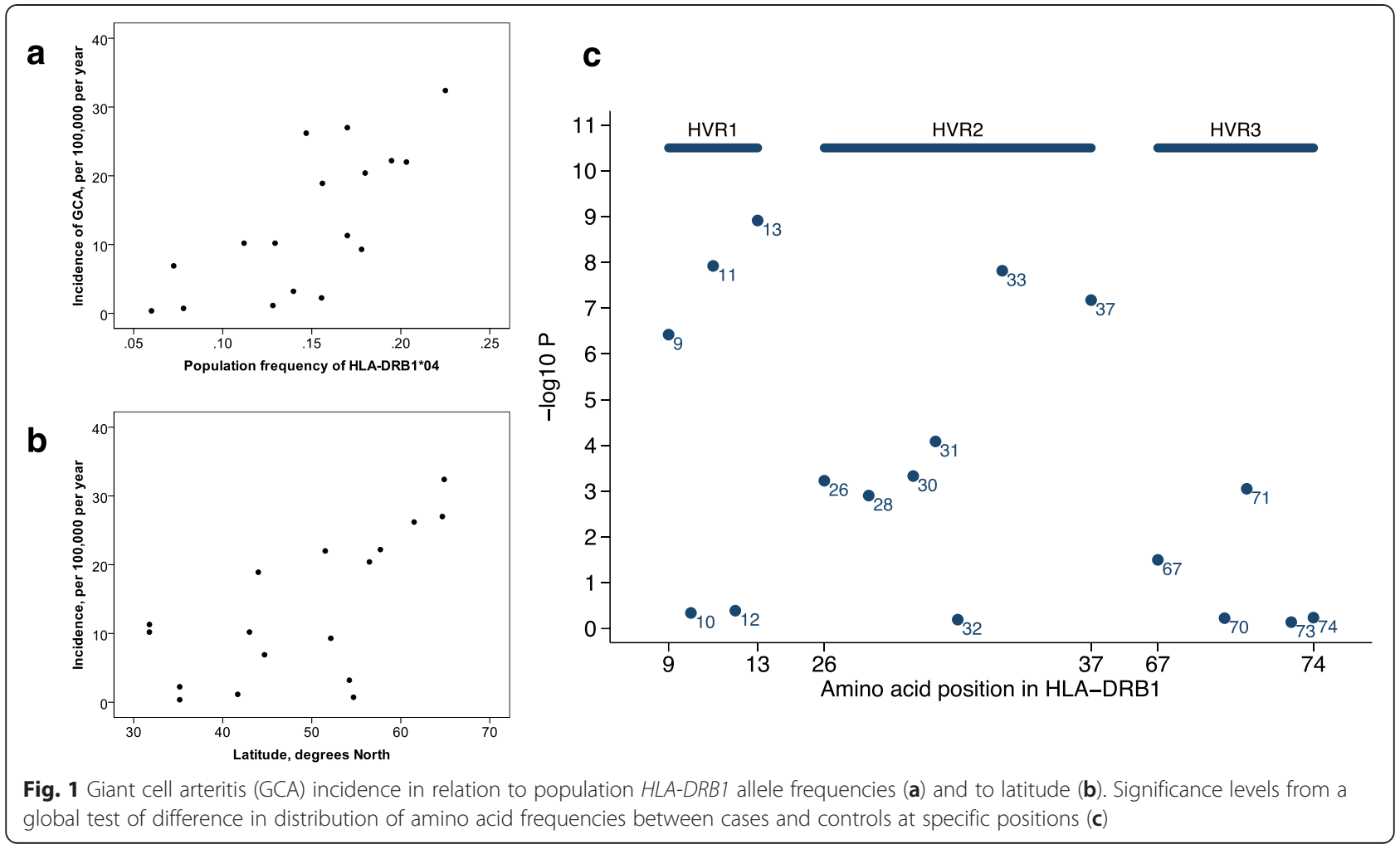

explained the observed HLA-DRB1 susceptibility and protective effects, rather than the previously proposed DRYF amino acid motif in the second hypervariable region [12]. We also observed that some non-HLA-DRB1*04 amino acid residues had additional effects (individual amino acid residues that were retained by a multivariable regression model for each separate amino acid position are shown in the last two columns in Table 5), suggesting additional genetic complexities that we did not have the power to investigate in depth. We then systematically extracted data on population prevalence of the identified susceptibility and protective $H L A-D R B 1$ alleles and compared this with reports of GCA incidence in different countries. We found a significant and independent relationship of GCA incidence both with $H L A-D R B 1 * 04$ and with latitude. Conversely, HLA-DRB1*15 was, if anything, protective and did not contribute to incidence of GCA in the geo-epidemiological study.

Strengths of this work include the presentation of the first UK HLA data in GCA, its presentation in the context of the international literature, the first meta-analysis of HLA-DRB1*04 GCA susceptibility studies, and the novel approach combining a traditional genetic association study with a geo-epidemiology approach. Using logistic regression for the UK, we could control for already-known $H L A-D R B 1$ susceptibility effects in the per-allele analysis, which also has not been performed in other datasets, which mostly reported only carrier frequencies not allele frequencies. Based on this, we were able to suggest HLA-DRB1 amino acid residues that best fit the observed susceptibility/protective allele effects. This is the first synthesis of the literature on reported GCA incidence in relation to population $H L A-D R B 1$ allele frequency and geographical latitude.

Our analysis is based on certain assumptions. Firstly, because many clinicians in the UK do not always request temporal artery biopsy except in cases of diagnostic doubt [24], we had prespecified in the analysis that GCA would be defined clinically rather than limiting inclusion to biopsy-positive cases only. The clinically diagnosed patients, however, had to be firmly diagnosed by an experienced consultant, and there had to be unequivocal clinical features and no alternative explanation for the symptoms after follow-up. Temporal artery biopsy is not $100 \%$ sensitive for GCA; possible reasons for falsenegative biopsies in our cohort included delays in obtaining biopsies resulting in resolution of inflammation, suboptimal biopsy length, and biopsy reporting based on the classic pathologic criteria, which may be overly stringent [25]; sometimes the temporal artery is spared in patients with GCA, particularly in those with predominant disease of the aorta and its proximal branches [26]. We conducted a sub-analysis of the biopsy-positive subset and found no difference in the observed effect size for $H L A$ - 
Table 5 Results of tests for association of amino acid at the most significant positions: 9, 11, 13, 33, and 37

\begin{tabular}{|c|c|c|c|c|c|c|c|}
\hline \multirow{2}{*}{$\begin{array}{l}\text { Position in } \\
\text { HLA-DRB1 }\end{array}$} & \multirow{2}{*}{$\begin{array}{l}\text { Amino } \\
\text { acid residue }\end{array}$} & \multicolumn{2}{|c|}{ Frequency } & \multicolumn{2}{|c|}{ Univariable } & \multicolumn{2}{|c|}{ Stepwise model (entry $P=0.05$ ) applied separately at each position } \\
\hline & & Cases & Controls & OR & $P$ value & OR & $P$ value \\
\hline \multirow[t]{3}{*}{9} & $\mathrm{E}$ & 0.698 & 0.565 & 1.78 & $7.3 \times 10^{-8}$ & & \\
\hline & K & 0.011 & 0.011 & 1.03 & 0.96 & & \\
\hline & W & 0.291 & 0.424 & 0.56 & $5.8 \times 10^{-8}$ & 0.56 & $5.85 \times 10^{-8}$ \\
\hline \multirow[t]{6}{*}{11} & $\mathrm{D}$ & 0.011 & 0.011 & 1.03 & 0.96 & & \\
\hline & G & 0.127 & 0.142 & 0.88 & 0.38 & & \\
\hline & $L$ & 0.060 & 0.123 & 0.46 & $3.6 \times 10^{-5}$ & 0.64 & 0.040 \\
\hline & $P$ & 0.104 & 0.159 & 0.63 & 0.0024 & & \\
\hline & $S$ & 0.391 & 0.371 & 1.09 & 0.41 & 1.37 & 0.015 \\
\hline & V & 0.307 & 0.194 & 1.86 & $1.4 \times 10^{-7}$ & 2.06 & $2.8 \times 10^{-7}$ \\
\hline \multirow[t]{6}{*}{13} & F & 0.071 & 0.141 & 0.48 & $1.7 \times 10^{-5}$ & 0.66 & 0.038 \\
\hline & G & 0.040 & 0.045 & 0.87 & 0.60 & & \\
\hline & $\mathrm{H}$ & 0.307 & 0.187 & 1.96 & $1.5 \times 10^{-8}$ & 2.11 & $5.5 \times 10^{-8}$ \\
\hline & $\mathrm{R}$ & 0.104 & 0.159 & 0.63 & 0.0024 & & \\
\hline & $S$ & 0.351 & 0.325 & 1.12 & 0.28 & 1.38 & 0.014 \\
\hline & Y & 0.127 & 0.142 & 0.88 & 0.38 & & \\
\hline \multirow[t]{2}{*}{33} & $\mathrm{H}$ & 0.307 & 0.187 & 1.96 & $1.5 \times 10^{-8}$ & 1.96 & $1.527 \times 10^{-8}$ \\
\hline & $N$ & 0.693 & 0.813 & 0.51 & $1.5 \times 10^{-8}$ & & \\
\hline \multirow[t]{5}{*}{37} & $F$ & 0.147 & 0.161 & 0.89 & 0.39 & & \\
\hline & $L$ & 0.022 & 0.017 & 1.27 & 0.49 & & \\
\hline & $\mathrm{N}$ & 0.256 & 0.219 & 1.09 & 0.46 & & \\
\hline & S & 0.164 & 0.282 & 0.51 & $8.0 \times 10^{-8}$ & 0.59 & 0.00012 \\
\hline & Y & 0.402 & 0.276 & 1.63 & $3.2 \times 10^{-6}$ & 1.37 & 0.0055 \\
\hline
\end{tabular}

Forward stepwise regression was used to determine whether there are independent effects of more than one amino acid residue at the same position. Only amino acids that were retained by the stepwise regression model are shown in the last two columns. Odds ratio (OR) of less than 1.0 indicates a protective effect, whereas OR of more than 1.0 indicates a susceptibility effect. Note the high level of linkage disequilibrium at this locus; Additional file 1 shows the amino acid residues within the three hypervariable regions of HLA-DRB1

$D R B 1 * 04$ association compared with the whole group; with such a small number of biopsy-negative cases, no meaningful statement can be made about the effect size in that group. Our meta-analysis showed that the effect size in the cohort overall was also comparable to that observed in previous reports, some of which included only biopsypositive cases. If not all the cases truly had GCA, this would have reduced the power of the study ("diluted out" the genetic association) but would have been highly unlikely to introduce artefactual genetic associations because the differential diagnosis of GCA is so wide. Similar pragmatic approaches to case definition for genetics studies, accepting a small, finite rate of misclassification in order to maximise recruitment, have been successfully used in other genetic association studies [27]. We also did not have the power to study whether there are differences in the effect size between regions of the UK, but regional variations in the incidence of diagnosed GCA have been described [28]; it remains unclear how far this is influenced by regional variations in population $H L A-D R B 1$ frequency [29].
In regard to the $H L A-D R B 1$ typing, it is recognised that HLA-DRB1 represents only a small part of the whole MHC and also that not having complete sequence-based four-digit typing may have resulted in some important information being missed. This study focused on HLA-DRB1 and we did not set out to analyse variation elsewhere in the MHC [30]. However, our finding that non-HLA-DRB1*04 residues also contributed significantly to GCA susceptibility/protection (Table 5) suggests that other alleles may also be involved. The MHC is a complex locus with extensive linkage disequilibrium, and an MHC-wide analysis requires larger datasets and specialised analysis methods. A concurrent international, collaborative large-scale genetic analysis of GCA (including samples from this study), using a different genotyping platform (Immunochip) with more extensive coverage of the HLA region [31], shows evidence of wider involvement of the MHC region while confirming the strong association with $D R B 1 \% 04$. Lastly, the literature reviews and metaanalyses are limited by the small number of studies in 
the literature, many of which were published some years ago, with corresponding variations in case ascertainment and in genotyping assays. Larger datasets using modern genotyping and statistical analysis methods will reveal further GCA susceptibility alleles within the whole $H L A$ locus and allow their pattern of linkage disequilibrium to be analysed.

The $P$ values reported here should be considered in the light of multiple testing, but owing to the a priori suggestion of HLA-DRB1*04 association and lack of consensus as to how to adjust for multiple testing at a multi-allelic locus where the different alleles are not independent of each other, we did not consider a Bonferroni correction to be appropriate here. Nevertheless, model over-fitting is a possibility, and it is essential that our findings be replicated in an independent dataset.

\section{Conclusions}

In summary, we report a novel approach to studying genetic influences of disease by combining traditional genetic association studies with geo-epidemiology methods that capitalise on publicly available data. Our new UK data and a synthesis of the published literature suggest that HLA$D R B 1 * 04$ might explain part of the observed geographical variation in GCA incidence. This is consistent with an autoimmune aetiology for GCA [32]. However, we found additional variation in susceptibility (Table 5) and incidence (Fig. 1a, b) that is not fully explained by HLA$D R B 1 * 04$ and is likely to relate to additional, unknown genetic and environmental factors. Previous studies of GCA have also demonstrated an association between $H L A-D R B 1 * 04$ and visual loss [33] and also with glucocorticoid resistance [34]. Of interest, in Japan (where $H L A-D R B 1^{*} 04$ population frequency is low), large-vessel vasculitis (Takayasu arteritis) is relatively more common than GCA. Takayasu arteritis was associated with alleles containing the 11-13-33 V-H-H motif ( $H L A-$ $D R B 1^{* 0405)}$ in a Turkish population but was not associated with another allele also containing $\mathrm{V}-\mathrm{H}-\mathrm{H}$ (HLA-DRB1*0401) in a European-American population; HLA-DRB1*1502, which was associated with Takayasu arteritis in both populations [35], does not contain the $\mathrm{V}-\mathrm{H}-\mathrm{H}$ motif. Very few patients in our dataset had large-vessel imaging, but genetic characterisation of the subset of GCA patients who have largevessel involvement or temporal artery sparing or both [26] would be of interest in future studies. From a clinical perspective, further study of well-phenotyped cohorts is required to determine whether $H L A-D R B 1^{*} 04$ may serve as a biomarker of pathophysiologically relevant phenotypic disease subsets in order to develop better risk stratification, prediction of response to glucocorticoids, and ultimately targeted therapies.

\section{Additional files}

Additional file 1: Appendices I and II (Membership of consortia). Description of contents: Names and institutional affiliations of members of the UK GCA Consortium (Appendix I) and the UKRAG Consortium (Appendix II)

Additional file 2: Amino acids at hypervariable regions (HVR) in susceptibility, protective, and neutral GCA alleles. Table showing the amino acid residues in the hypervariable regions of HLA-DRB 1, for each of the HLA-DRB1 alleles. Shaded columns denote the proposed 11-13-33 GCA risk motif.

\section{Abbreviations}

ACR: American College of Rheumatology; Cl: Confidence interval; GCA: Giant cell arteritis; HLA: Human leukocyte antigen; HVR: Hypervariable region; MHC: Major histocompatibility complex; OR: Odds ratio; RA: Rheumatoid arthritis; UKRAG: UK Rheumatoid Arthritis Genetics.

\section{Competing interests}

The authors declare that they have no competing interests.

\section{Authors' contributions}

SLM designed the study and wrote the case record form, recruited patients, and validated clinical data, including diagnoses, DNA extractions, and genotyping assays, and performed the searches and data extraction from the published literature and online databases for HLA-DRB1 data (GCA

susceptibility, amino acid sequences, and population allele frequencies), participated in design of geo-epidemiology analyses and carried out the geo-epidemiology statistical analyses, and drafted the manuscript. JCT carried out all the other statistical analyses, including amino acid analyses, and helped to draft the manuscript. LH-R and SM participated in design and validation of the genotyping assays, including sequencing and analysis of electropherograms, including use of HaploViewer. BD, AG, MG, LH, SJ, and CTP recruited patients and validated clinical data, including diagnoses. UKRAG Consortium provided control HLA-DRB1 data. JHB supervised the statistical analyses, including amino acid analyses, and helped to draft the manuscript. RW supervised the design and conduct of the geo-epidemiology analyses, performed the literature search for incidence of GCA in different countries, and helped to draft the manuscript. AWM conceived of the study, supervised the design and conduct of the genetic studies, including amino acid analyses, and helped to draft the manuscript. All authors revised the draft for important intellectual content and read and approved the final manuscript.

\section{Acknowledgements}

Earlier versions of this work, including the proposal about the contribution of the HVR1 region, were presented at the UKIVAS Lockwood Club in Manchester, May 2013, and at the Northern and Yorkshire Combined Regional Meeting in York, September 2013; many audience members provided constructive feedback and comments at that time. Membership of the UK GCA Consortium at the time these patients were recruited [17] and UKRAG Consortium [18] can be found in the references cited and in

Additional file 2. We are grateful to all those who recruited patients at these sites, including Shouma Dutta and Jane Hollywood. We would like to thank lan Carr for writing the HaplotypeViewer program, specifically for this study. Thanks to Clive Edelsten, ophthalmologist, Ipswich Hospital, for discussions about Scandinavian contributions to UK ancestry.

This work was supported by the Wellcome Trust/Academy of Medical Sciences Starter Grants for Clinical Lecturers scheme. SLM was funded by a Clinical Research Fellowship from Research into Ageing, then a Clinical Lectureship from the National Institute for Health Research (NIHR), and is currently funded by a Clinician Scientist Award from the NIHR. AWM, JCT, and JHB are supported in part by the NIHR-Leeds Musculoskeletal Biomedical Research Unit. The views expressed are those of the authors and not necessarily those of the National Health Service, the NIHR, or the Department of Health.

\section{Grant funding}

Grant funding was provided by the NIHR and the Wellcome Trust. 


\section{Author details}

'School of Medicine and NIHR-Leeds Biomedical Research Unit, Chapel Allerton Hospital, Leeds LS7 4SA, West Yorkshire, UK. '2Department of Rheumatology, Southend University Hospital, Prittlewell Chase, Southend SSO ORY, Essex, UK. ${ }^{3}$ Department of Rheumatology, Harrogate and District Foundation NHS Trust, Lancaster Park Road, Harrogate HG2 7SX, North Yorkshire, UK. ${ }^{4}$ Department of Rheumatology, York Teaching Hospital NHS Foundation Trust, Wigginton Road, York YO31 8HE, North Yorkshire, UK. ${ }^{5}$ Department of Rheumatology, Dewsbury and District Hospital, Halifax Road, Dewsbury WF13 4HS, West Yorkshire, UK. ${ }^{6}$ Department of Rheumatology, Pinderfields General Hospital, Aberford Road, Wakefield WF1 4DG, West Yorkshire, UK. 7Department of Rheumatology, Chapel Allerton Hospital, Leeds, Leeds LS7 4SA, West Yorkshire, UK. ' Department of Rheumatology, Ipswich Hospital NHS Trust, Heath Road, Ipswich IP4 5PD, Suffolk, UK. 'Wellcome Trust Brenner Building, St. James's University Hospital, Beckett Street, Leeds LS9 7TF, West Yorkshire, UK

Received: 24 December 2014 Accepted: 18 June 2015

Published online: 30 July 2015

\section{References}

1. Padyukov L, Seielstad M, Ong RT, Ding B, Ronnelid J, Seddighzadeh M, et al. A genome-wide association study suggests contrasting associations in ACPA-positive versus ACPA-negative rheumatoid arthritis. Ann Rheum Dis. 2011;70:259-65.

2. Lyons PA, Rayner TF, Trivedi S, Holle JU, Watts RA, Jayne DR, et al. Genetically distinct subsets within ANCA-associated vasculitis. N Engl J Med. 2012;367:214-23

3. Gregersen PK, Silver J, Winchester RJ. The shared epitope hypothesis. An approach to understanding the molecular genetics of susceptibility to rheumatoid arthritis. Arthritis Rheum. 1987:30:1205-13.

4. Mackie SL, Taylor JC, Martin SG, Wordsworth P, Steer S, Wilson AG, et al. A spectrum of susceptibility to rheumatoid arthritis within HLA-DRB1: stratification by autoantibody status in a large UK population. Genes Immun. 2012;13:120-8.

5. Raychaudhuri S, Sandor C, Stahl EA, Freudenberg J, Lee HS, Jia X, et al. Five amino acids in three HLA proteins explain most of the association between MHC and seropositive rheumatoid arthritis. Nat Genet. 2012:44:291-6.

6. Gran JT. Some thoughts about the etiopathogenesis of temporal arteritis-a review. Scand J Rheumatol. 2002;31:1-5.

7. Gabriel SE, Michaud K. Epidemiological studies in incidence, prevalence, mortality, and comorbidity of the rheumatic diseases. Arthritis Res Ther. 2009:11:229.

8. Watts RA, Scott DG. Epidemiology of vasculitis. In: Ball GV, Fessler BJ, Bridges Jr SL, editors. Oxford Textbook of Vasculitis. 3rd ed. Oxford: Oxford University Press; 2014. p. 7-25.

9. Ball GV, Fessler BJ, Bridges Jr SL. Oxford Textbook of Vasculitis. 3rd ed. Oxford: Oxford University Press; 2014.

10. Rooney PJ, Rooney J, Balint G, Balint P. Polymyalgia rheumatica: 125 years of progress? Scott Med J. 2014;59:220-8.

11. Gonzalez-Gay MA, Amoli MM, Garcia-Porrua C, Ollier WE. Genetic markers of disease susceptibility and severity in giant cell arteritis and polymyalgia rheumatica. Semin Arthritis Rheum. 2003;33:38-48.

12. Weyand CM, Hicok KC, Hunder GG, Goronzy JJ. The HLA-DRB1 locus as a genetic component in giant cell arteritis. Mapping of a disease-linked sequence motif to the antigen binding site of the HLA-DR molecule. J Clin Invest. 1992;90:2355-61.

13. Martinez-Taboda VM, Bartolome MJ, Lopez-Hoyos M, Blanco R, Mata C, Calvo J, et al. HLA-DRB1 allele distribution in polymyalgia rheumatica and giant cell arteritis: influence on clinical subgroups and prognosis. Semin Arthritis Rheum. 2004;34:454-64.

14. Heath SC, Gut IG, Brennan P, McKay JD, Bencko V, Fabianova E, et al. Investigation of the fine structure of European populations with applications to disease association studies. Eur J Hum Genet. 2008;16:1413-29.

15. Hunder GG, Bloch DA, Michel BA, Stevens MB, Arend WP, Calabrese LH, et al. The American College of Rheumatology 1990 criteria for the classification of giant cell arteritis. Arthritis Rheum. 1990:33:1122-8.

16. Rao JK, Allen NB, Pincus T. Limitations of the 1990 American College of Rheumatology classification criteria in the diagnosis of vasculitis. Ann Intern Med. 1998;129:345-52

17. Mackie SL, Dasgupta B, Hordon L, Gough A, Green M, Hollywood J, Dutta S, Bejarano V, Jarrett S, Morgan AW et al. Ischaemic manifestations in giant cell arteritis are associated with area level socio-economic deprivation, but not cardiovascular risk factors. Rheumatology (Oxford, England). 2011;50:2014-22.

18. Morgan AW, Thomson W, Martin SG, Carter AM, Erlich HA, Barton A, et al. Reevaluation of the interaction between HLA-DRB1 shared epitope alleles, PTPN22, and smoking in determining susceptibility to autoantibody-positive and autoantibody-negative rheumatoid arthritis in a large UK Caucasian population. Arthritis Rheum. 2009;60:2565-76.

19. Carr I. HaplotypeViewer. http://dna.leeds.ac.uk/HaplotypeViewer/. Accessed 17 Dec 2013.

20. DerSimonian R, Laird N. Meta-analysis in clinical trials. Control Clin Trials. 1986;7:177-88

21. Watts RA, Mackie SL, MacGregor AJ. HLA allele variation as a potential explanation for the geographical distribution of granulomatosis with polyangiitis. Rheumatology (Oxford). 2015;54:359-62.

22. Gonzalez-Galarza FF, Christmas S, Middleton D, Jones AR. Allele frequency net: a database and online repository for immune gene frequencies in worldwide populations. Nucleic Acids Res. 2011;39:D913-9.

23. Robinson J, Mistry K, McWilliam H, Lopez R, Parham P, Marsh SGE. The IMGT/HLA Database. Nucleic Acids Res. 2011;39(Suppl 1):D1171-6. http://www.ebi.ac.uk/ipd/imgt/hla. Accessed 31 Jan 2012.

24. Davies C, Frost B, Eshan O, McLain AD, Shandall A. Temporal artery biopsy.. who needs one? Postgrad Med J. 2006;82:476-8.

25. Zhou L, Luneau K, Weyand CM, Biousse V, Newman NJ, Grossniklaus HE. Clinicopathologic correlations in giant cell arteritis: a retrospective study of 107 cases. Ophthalmology. 2009;116:1574-80.

26. Brack A, Martinez-Taboada V, Stanson A, Goronzy JJ, Weyand CM. Disease pattern in cranial and large-vessel giant cell arteritis. Arthritis Rheum. 1999;42:311-7.

27. Genome-wide association study of 14,000 cases of seven common diseases and 3,000 shared controls. Nature. 2007:447:661-78.

28. Smeeth L, Cook C, Hall AJ. Incidence of diagnosed polymyalgia rheumatica and temporal arteritis in the United Kingdom, 1990-2001. Ann Rheum Dis. 2006:65:1093-8.

29. Winney B, Boumertit A, Day T, Davison D, Echeta C, Evseeva I, et al. People of the British Isles: preliminary analysis of genotypes and surnames in a UK-control population. Eur J Hum Genet. 2012;20:203-10.

30. Gonzalez-Gay MA, Rueda B, Vilchez JR, Lopez-Nevot MA, Robledo G, Ruiz MP, et al. Contribution of MHC class I region to genetic susceptibility for giant cell arteritis. Rheumatology (Oxford, England). 2007;46:431-34.

31. Carmona FD, Mackie SL, Martin J-E, Taylor JC, Vaglio A, Eyre S, et al. A large-scale genetic analysis reveals a strong contribution of the HLA Class II region to giant cell arteritis susceptibility. Am J Hum Genet. 2015;96:565-80.

32. Weyand CM, Schonberger J, Oppitz U, Hunder NN, Hicok KC, Goronzy JJ. Distinct vascular lesions in giant cell arteritis share identical T cell clonotypes. J Exp Med. 1994;179:951-60.

33. Gonzalez-Gay MA, Garcia-Porrua C, Llorca J, Hajeer AH, Branas F, Dababneh A, et al. Visual manifestations of giant cell arteritis. Trends and clinical spectrum in 161 patients. Medicine. 2000;79:283-92.

34. Rauzy O, Fort M, Nourhashemi F, Alric L, Juchet $H$, Ecoiffier M, et al. Relation between HLA DRB1 alleles and corticosteroid resistance in giant cell arteritis. Ann Rheum Dis. 1998;57:380-2.

35. Saruhan-Direskeneli G, Hughes T, Aksu K, Keser G, Coit P, Aydin SZ, et al. Identification of multiple genetic susceptibility loci in Takayasu arteritis. Am J Hum Genet. 2013;93:298-305.

36. Armstrong RD, Panayi GS, Welsh KI. Polymyalgia rheumatica and rheumatoid arthritis: similarity of HLA antigen frequencies. Arthritis Rheum. 1984;27:1438-9.

37. Bignon JD, Barrier J, Soulillou JP, Martin P, Grolleau JY. HLA DR4 and giant cell arteritis. Tissue Antigens. 1984;24:60-2.

38. Calamia KT, Moore SB, Elveback LR, Hunder GG. HLA-DR locus antigens in polymyalgia rheumatica and giant cell arteritis. J Rheumatol. 1981;8:993-6.

39. Cid MC, Ercilla G, Vilaseca J, Sanmarti R, Villalta J, Ingelmo M, et al. Polymyalgia rheumatica: a syndrome associated with HLA-DR4 antigen. Arthritis Rheum. 1988;31:678-82.

40. Combe B, Sany J, Le Quellec A, Clot J, Eliaou JF. Distribution of HLA-DRB1 alleles of patients with polymyalgia rheumatica and giant cell arteritis in a Mediterranean population. J Rheumatol. 1998;25:94-8.

41. Dababneh A, Gonzalez-Gay MA, Garcia-Porrua C, Hajeer A, Thomson W, Ollier W. Giant cell arteritis and polymyalgia rheumatica can be differentiated by distinct patterns of HLA class II association. J Rheumatol. 1998:25:2140-5.

42. Gouet D, Alcalay D, Azais I, Alcalay M, Becq-Giraudon B, Sudre Y, et al. HLA-DR antigens in polymyalgia rheumatica and giant cell arteritis. J Rheumatol. 1985;12:627-8. 
43. Hansen JA, Healey LA, Wilske KR. Association between giant cell (temporal) arteritis and HLA-Cw3. Hum Immunol. 1985;13:193-8.

44. Jacobsen S, Baslund B, Madsen HO, Tvede N, Svejgaard A, Garred P. Mannose-binding lectin variant alleles and HLA-DR4 alleles are associated with giant cell arteritis. J Rheumatol. 2002;29:2148-53.

45. Ninet J, Gebuhrer L, Bonvoisin B, Mackiewicz R, Laurent H, Boussuge C, Rousset H, Le Petit JC, Betuel H. [Distribution of HLA-DR antigens in unrelated giant cell arteritis]. Presse medicale (Paris, France : 1983). 1987;16:1725-28.

46. Richardson JE, Gladman DD, Fam A, Keystone EC. HLA-DR4 in giant cell arteritis: association with polymyalgia rheumatica syndrome. Arthritis Rheum. 1987:30:1293-7.

47. Salvarani C, Boiardi L, Mantovani V, Ranzi A, Cantini F, Olivieri I, et al. HLA-DRB1, DQA1, and DQB1 alleles associated with giant cell arteritis in northern Italy. J Rheumatol. 1999;26:2395-9.

48. Weyand CM, Hunder NN, Hicok KC, Hunder GG, Goronzy JJ. HLA-DRB1 alleles in polymyalgia rheumatica, giant cell arteritis, and rheumatoid arthritis. Arthritis Rheum. 1994;37:514-20.

49. Franzen $P$, Sutinen $S$, von Knorring J. Giant cell arteritis and polymyalgia rheumatica in a region of Finland: an epidemiologic, clinical and pathologic study, 1984-1988. J Rheumatol. 1992;19:273-6.

50. Haimila K, Perasaari J, Linjama T, Koskela S, Saarinenl T, Lauronen J, et al. HLA antigen, allele and haplotype frequencies and their use in virtual panel reactive antigen calculations in the Finnish population. Tissue Antigens. 2013;81:35-43.

51. Nordborg C, Johansson H, Petursdottir $V$, Nordborg E. The epidemiology of biopsy-positive giant cell arteritis: special reference to changes in the age of the population. Rheumatology (Oxford, England). 2003;42:549-52.

52. Grunewald J, Brynedal B, Darlington P, Nisell M, Cederlund K, Hillert J, et al. Different HLA-DRB1 allele distributions in distinct clinical subgroups of sarcoidosis patients. Respir Res. 2010;11:25.

53. Hedstrom AK, Sundqvist E, Baarnhielm M, Nordin N, Hillert J, Kockum I, et al. Smoking and two human leukocyte antigen genes interact to increase the risk for multiple sclerosis. Brain. 2011;134:653-64.

54. Bjorkbacka H, Lavant EH, Fredrikson GN, Melander O, Berglund G, Carlson $J A$, et al. Weak associations between human leucocyte antigen genotype and acute myocardial infarction. J Intern Med. 2010;268:50-8.

55. van der Woude D, Lie BA, Lundstrom E, Balsa A, Feitsma AL, HouwingDuistermaat JJ, et al. Protection against anti-citrullinated protein antibodypositive rheumatoid arthritis is predominantly associated with HLA-DRB1*1301: a meta-analysis of HLA-DRB1 associations with anti-citrullinated protein antibody-positive and anti-citrullinated protein antibody-negative rheumatoid arthritis in four European populations. Arthritis Rheum. 2010;62:1236-45.

56. Castro FA, Haimila K, Sareneva I, Schmitt M, Lorenzo J, Kunkel N, et al. Association of HLA-DRB1, interleukin-6 and cyclin D1 polymorphisms with cervical cancer in the Swedish population-a candidate gene approach. Int J Canc Suppl J Int Canc Suppl. 2009;125:1851-8.

57. Salvarani C, Macchioni P, Zizzi F, Mantovani W, Rossi F, Castri C, et al. Epidemiologic and immunogenetic aspects of polymyalgia rheumatica and giant cell arteritis in northern Italy. Arthritis Rheum. 1991;34:351-6.

58. Rendine S, Ferrero NM, Sacchi N, Costa C, Pollichieni S, Amoroso A. Estimation of human leukocyte antigen class I and class II high-resolution allele and haplotype frequencies in the Italian population and comparison with other European populations. Hum Immunol. 2012;73:399-404.

59. Invernizzi $P$, Selmi C, Poli F, Frison S, Floreani A, Alvaro D, Almasio P, Rosina F, Marzioni M, Fabris L et al. Human leukocyte antigen polymorphisms in Italian primary biliary cirrhosis: a multicenter study of 664 patients and 1992 healthy controls. Hepatology (Baltimore, Md). 2008:48:1906-12.

60. Gonzalez-Gay MA, Garcia-Porrua C, Rivas MJ, Rodriguez-Ledo P, Llorca J. Epidemiology of biopsy proven giant cell arteritis in northwestern Spain: trend over an 18 year period. Ann Rheum Dis. 2001;60:367-71.

61. Alcoceba M, Marin L, Balanzategui A, Sarasquete ME, Chillon MC, MartinJimenez $P$, et al. Frequency of HLA-A, $-B$ and -DRB1 specificities and haplotypic associations in the population of Castilla y Leon (northwest-central Spain). Tissue Antigens. 2011;78:249-55.

62. Romero-Pinel L, Pujal JM, Martinez-Yelamos S, Gubieras L, Matas E, Bau L, et al. HLA-DRB1: genetic susceptibility and disability progression in a Spanish multiple sclerosis population. Eur J Neurol. 2011;18:337-42.

63. Elling $P$, Olsson AT, Elling $H$. Synchronous variations of the incidence of temporal arteritis and polymyalgia rheumatica in different regions of Denmark; association with epidemics of Mycoplasma pneumoniae infection. J Rheumatol. 1996:23:112-9.
64. Kruse C, Steffensen R, Varming K, Christiansen OB. A study of HLA-DR and -DQ alleles in 588 patients and 562 controls confirms that HLA-DRB ${ }^{*} 03$ is associated with recurrent miscarriage. Human reproduction (Oxford, England). 2004;19:1215-21.

65. Baldursson O, Steinsson K, Bjornsson J, Lie JT. Giant cell arteritis in Iceland. An epidemiologic and histopathologic analysis. Arthritis Rheum. 1994;37:1007-12.

66. Jonsson T, Gudmundsson KG, Bjarnadottir K, Hjalmarsdotti IB, Gudmundsson S, Arngrimsson R. Association of HLA-DRB1*01 with Dupuytren's disease. Scand J Rheumatol. 2013;42:45-7.

67. Haugeberg G, Irgens KA, Thomsen RS. No major differences in incidence of temporal arteritis in northern and western Norway compared with reports from southern Norway. Scand J Rheumatol. 2003;32:318-9.

68. Munthe-Kaas MC, Carlsen KL, Carlsen KH, Egeland T, Haland G, Devulapalli CS, et al. HLA Dr-Dq haplotypes and the TNFA-308 polymorphism: associations with asthma and allergy. Allergy. 2007;62:991-8.

69. Karlsen TH, Boberg KM, Vatn M, Bergquist A, Hampe J, Schrumpf E, et al. Different HLA class II associations in ulcerative colitis patients with and without primary sclerosing cholangitis. Genes Immun. 2007;8:275-8.

70. Reinhold-Keller E, Herlyn K, Wagner-Bastmeyer R, Gross WL. Stable incidence of primary systemic vasculitides over five years: results from the German vasculitis register. Arthritis Rheum. 2005;53:93-9.

71. Reinshagen M, Loeliger C, Kuehnl P, Weiss U, Manfras BJ, Adler G, et al. HLA class II gene frequencies in Crohn's disease: a population based analysis in Germany. Gut. 1996:38:538-42.

72. Dadoniene J, Kirdaite G, Mackiewicz Z, Rimkevicius A, Haugeberg G. Incidence of primary systemic vasculitides in Vilnius: a university hospital population based study. Ann Rheum Dis. 2005;64:335-6.

73. Ploski R, Butrimiene I, Kaminska E, Valiukiene K, Sliwinska P, Kubasiewicz E, et al. Rheumatoid arthritis in Poland and Lithuania: different clinical course and HLA associations despite similar genetic background. Ann Rheum Dis. 2005;64:165-6.

74. Bas-Lando M, Breuer GS, Berkun Y, Mates M, Sonnenblick M, Nesher G. The incidence of giant cell arteritis in Jerusalem over a 25-year period: annual and seasonal fluctuations. Clin Exp Rheumatol. 2007;25:S15-7.

75. Roitberg-Tambur A, Witt CS, Friedmann A, Safirman C, Sherman L, Battat S, et al. Comparative analysis of HLA polymorphism at the serologic and molecular level in Moroccan and Ashkenazi Jews. Tissue Antigens. 1995;46:104-10.

76. Sonnenblick M, Nesher G, Friedlander Y, Rubinow A. Giant cell arteritis in Jerusalem: a 12-year epidemiological study. Br J Rheumatol. 1994;33:938-41.

77. Kwon OJ, Brautbar C, Weintrob N, Sprecher E, Saphirman C, Bloch K, et al. Immunogenetics of HLA class II in Israeli Ashkenazi Jewish, Israeli non-Ashkenazi Jewish, and in Israeli Arab IDDM patients. Hum Immunol. 2001;62:85-91.

78. Zeitlin AA, Heward JM, Newby PR, Carr-Smith JD, Franklyn JA, Gough SC, et al. Analysis of HLA class II genes in Hashimoto's thyroiditis reveals differences compared to Graves' disease. Genes Immun. 2008;9:358-63.

79. Pamuk ON, Donmez S, Karahan B, Pamuk GE, Cakir N. Giant cell arteritis and polymyalgia rheumatica in northwestern Turkey: Clinical features and epidemiological data. Clin Exp Rheumatol. 2009;27:830-3.

80. Ucar F, Capkin E, Karkucak M, Yucel B, Sonmez M, Alver A, et al. Associations of HLA-DRB1 alleles with anti-citrullinated protein antibody-positive and anti-citrullinated protein antibody-negative rheumatoid arthritis in northern east part of Turkey. Int J Rheum Dis. 2012;15:538-45.

81. Saruhan-Direskeneli G, Uyar FA, Bakar S, Eraksoy M. Molecular analysis of HLA-DRB1, -DQA1 and -DQB1 polymorphism in Turkey. Tissue Antigens. 2000;55:171-4

82. Kinikli G, Ates A, Turgay M, Akay G, Kinikli S, Tokgoz G. HLA-DRB1 genes and disease severity in rheumatoid arthritis in Turkey. Scand J Rheumatol. 2003:32:277-80.

83. Kermani TA, Schafer VS, Crowson CS, Hunder GG, Gabriel SE, Matteson EL, et al. Increase in age at onset of giant cell arteritis: a population-based study. Ann Rheum Dis. 2010;69:780-1.

84. Smith CA, Fidler WJ, Pinals RS. The epidemiology of giant cell arteritis. Report of a ten-year study in Shelby County, Tennessee. Arthritis Rheum. 1983;26:1214-9.

85. Leech NJ, Kitabchi AE, Gaur LK, Hagopian WA, Hansen J, Burghen GA, et al. Genetic and immunological markers of insulin dependent diabetes in Black Americans. Autoimmunity. 1995;22:27-32.

86. Ramstead CL, Patel AD. Giant cell arteritis in a neuro-ophthalmology clinic in Saskatoon, 1998-2003. Can J Ophthalmol. 2007:42:295-8. 
87. Gladman DD, Kung TN, Siannis F, Pellett F, Farewell VT, Lee P. HLA markers for susceptibility and expression in scleroderma. J Rheumatol. 2005;32:1481-7.

88. Silverberg MS, Mirea L, Bull SB, Murphy JE, Steinhart AH, Greenberg GR, et al. A population- and family-based study of Canadian families reveals association of HLA DRB1*0103 with colonic involvement in inflammatory bowel disease. Inflamm Bowel Dis. 2003;9:1-9.

Submit your next manuscript to BioMed Central and take full advantage of:

- Convenient online submission

- Thorough peer review

- No space constraints or color figure charges

- Immediate publication on acceptance

- Inclusion in PubMed, CAS, Scopus and Google Scholar

- Research which is freely available for redistribution 